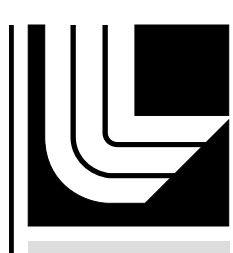

LA W RENCE LIVERMORE NATION AL LABORATORY

\title{
Growth of deformation twins in Tantalum via coherent twin boundary migration
}

L. A. Sandoval, M. P. Surh, A. A. Chernov, D. F. Richards

June 13, 2013

Journal of Applied Physics 
This document was prepared as an account of work sponsored by an agency of the United States government. Neither the United States government nor Lawrence Livermore National Security, LLC, nor any of their employees makes any warranty, expressed or implied, or assumes any legal liability or responsibility for the accuracy, completeness, or usefulness of any information, apparatus, product, or process disclosed, or represents that its use would not infringe privately owned rights. Reference herein to any specific commercial product, process, or service by trade name, trademark, manufacturer, or otherwise does not necessarily constitute or imply its endorsement, recommendation, or favoring by the United States government or Lawrence Livermore National Security, LLC. The views and opinions of authors expressed herein do not necessarily state or reflect those of the United States government or Lawrence Livermore National Security, LLC, and shall not be used for advertising or product endorsement purposes. 


\title{
Growth of deformation twins in Tantalum via coherent twin boundary migration
}

\author{
Luis A. Sandoval,* Michael P. Surh, Alexander A. Chernov, and David F. Richards \\ Condensed Matter and Materials, \\ Lawrence Livermore National Laboratory, \\ Livermore, CA 94550, USA
}

\begin{abstract}
In order to investigate the process of twinning growth, the nucleation of twinning dislocation loops on the coherent boundary of a Tantalum twin superlattice is studied via a molecular dynamics approach. We get homogeneous nucleation rates by means of a stationarity test and the mean first-passage method. We study their dependence on driving force and temperature in a framework given by the Kolgomorov-Johnson-Mehl-Avrami (KJMA) theory. Correspondingly, the contribution to the twinning growth dictated by this nucleation mechanism, as compared to the pole mechanism, is discussed. The homogeneous loop nucleation and growth mechanism can be relevant to twinning in high strain-rate experiments as in shock waves.
\end{abstract}

\section{INTRODUCTION}

Twinning is one of the main modes of plastic deformation in crystals, being particularly important under conditions of low temperature or high strain rates. It is also a major feature observed in many martensitic transformations, recrystallization processes and crystal growth from vapour or liquid phases ${ }^{1,2}$. In plastic deformation, the time-dependent volume of twinning contributes to the overall strain simply according to the eigenstrain of the twin transformation. However despite decades of work there are still many open questions on the role of twinning in constitutive behavior, especially in bcc metals.

The complete process of mechanical (deformation) twinning is divided into nucleation and growth stages. Computational and experimental evidence suggests that twins nucleate via a cooperative emission of partials thanks to the presence of defects which concentrate the stress required for the process ${ }^{3-5}$. After nucleation, the subsequent growth of twin embryos requires stresses considerably lower than those needed for twin nucleation. Growth is strongly anisotropic due to the competing role of different mechanisms. The emitted twinning partials form highly mobile incoherent twin boundaries ${ }^{1,6}$, which commonly extend until they encounter obstacles like grain boundaries or other interfaces. The result is the formation of thin twin lamellae bounded by coherent twin interfaces. Further twin thickening requires other mechanisms to form new layers at the coherent boundaries.

Twin thickening has been proposed to occur by multiple processes. One of them is the pole mechanism, which describes the glide of twinning partials around a screw dislocation in a manner resembling the Frank mechanism of spiral crystal growth. Another possible mechanism of twin growth is the 2d-nucleation and growth of (possibly multiple) closed twinning dislocation loops on the coherent twin boundary. (A related atomistic and continuum theory study of the homogeneous nucleation of isolated, perfect dislocation loops has been recently been published ${ }^{7-9}$.) This second mechanism can operate in the absence of additional defects to act as a pole, e.g., in nearly perfect crystals. The contribution from this mechanism has been previously studied in detail ${ }^{1,2}$, after considering that the interface region may have distinct elastic properties than the bulk. This is a standard growth problem that can be treated by the Kolgomorov-JohnsonMehl-Avrami (KJMA) theory ${ }^{10-12}$. We apply atomistic simulations to obtain the required nucleation and growth rate parameters versus temperature and stress for this more continuum-like model.

The total free energy change due to the formation of an elliptic twin dislocation loop is given by

$$
\begin{aligned}
\Delta G & =-n \Delta \mu+c \gamma_{d} \\
& =-\frac{\pi a^{2} \sqrt{1-e^{2}} h}{\omega} \Delta \mu+4 a E(e) \gamma_{d},
\end{aligned}
$$

where $n$ is the number of atoms which belong to the $2 \mathrm{~d}$ nucleus, $\Delta \mu$ is the driving force, $c$ is the loop's circumference, $\gamma_{d}$ is the line energy density, $a$ is the loop's semi-major axis, $e$ denotes the ellipse's eccentricity, $\omega$ denotes the atomic volume, $h$ corresponds to the step's height (distance between ( $2 \overline{1} 1)$ planes), and $E$ is the complete elliptic integral of the second kind ${ }^{13}$. The generalized driving force is given by $\Delta \mu=\omega \sigma_{x y} \epsilon_{0}$, that is, it depends on the average acting stress $\sigma_{x y}$ times the elementary twinning strain $\epsilon_{0}=1 / \sqrt{2}$, corresponding to $(2 \overline{1} 1)[11 \overline{1}]$ twinning in bcc metals ${ }^{2}$. Assuming a constant (size-independent) eccentricity, the critical semi-major axis and activation free energy are given, respectively, by

$$
a=\frac{\omega \gamma_{d}}{h \Delta \mu} \frac{2 E(e)}{\pi \sqrt{1-e^{2}}},
$$

and

$$
\Delta G_{c}=\frac{\omega \gamma_{d}^{2}}{h \Delta \mu} \frac{4 E^{2}(e)}{\pi \sqrt{1-e^{2}}} .
$$

The nucleation rate of twinning dislocation loops on the interface of a coherent twin boundary, $J_{2 d}$, is expressed by an Arrhenius-type equation 


$$
J_{2 d}=B \exp \left(-\frac{\Delta G_{c}}{k T}\right) .
$$

For simplicity let us consider that, once nucleated, the dislocation loop spreads rapidly with a front velocity $v_{t}$ in all directions on the plane (although, actually, the edge and screw components move at different speeds). From KJMA theory the average time required to complete onelayer twin boundary migration is $\sim\left(J_{2 d} v_{t}^{2}\right)^{-1 / 3}$, therefore the growth rate normal to the coherent twin boundary (or velocity of twin thickening) is given by ${ }^{14}$

$$
v_{n} \sim h\left(J_{2 d} v_{t}^{2}\right)^{1 / 3} .
$$

In this work we focus our attention in the calculation of nucleation rates using either a simple average time to nucleation or the mean first-passage times (MFPT) method ${ }^{15}$ which considers the clustering of displaced atoms inside the partial dislocation loop. We combine this with estimates of the partial dislocation velocity to compare the importance of pole and nucleation/growth mechanisms under typical experimental conditions. More details of the dislocation stress-velocity relation and dislocation-dislocation reactions for twinning partials will be considered in a subsequent study. As expected, the importance of the nucleation and growth process relative to the pole mechanism increases at higher stresses. It is expected make significant contributions to twinning growth in Ta under experimentally achievable conditions.

\section{METHODOLOGY}

Deformation twins are formed in bcc on (2111) planes with a shear strain of $1 / \sqrt{2}$ in the [111] direction. Our simulation cell consists of 2021760 atoms in a layered (twinned) bcc crystal with dimensions $60[11 \overline{1}] \times 39[2 \overline{1} 1] \times$ $72[0 \overline{1} \overline{1}]$ (about $34.4 \times 31.6 \times 33.7 \mathrm{~nm}^{3}$ at $0 \mathrm{~K}$ and $0 \mathrm{GPa}$ ), with periodic boundary conditions in all directions (see the inset of Fig. 1 (a)). The dynamics is determined by an improved Finnis-Sinclair potential for $\mathrm{Ta}^{16}$. Before performing nucleation simulations, we studied the atomic structure of the $(2 \overline{1} 1)$ twin boundary in order to find the minimum energy interface. Starting from an ideal, mirror-symmetric twin boundary we changed the relative location of the grains by translating one of them across the $(2 \overline{1} 1)$ twin boundary. After this simple minimization process (rigid grain translation without relaxation) we found that the minimum energy interface corresponds to an isosceles twin boundary ${ }^{2,17}$, as has been predicted from ab initio calculations with full relaxation ${ }^{18}$, which basically consists of a $\frac{1}{12}[11 \overline{1}]$ shift of one grain relative to the other one. Subsequently the system was equilibrated during $50 \mathrm{ps}$ at the desired temperature via a Langevin thermostat with decay time of 0.1 ps. Additionally, for

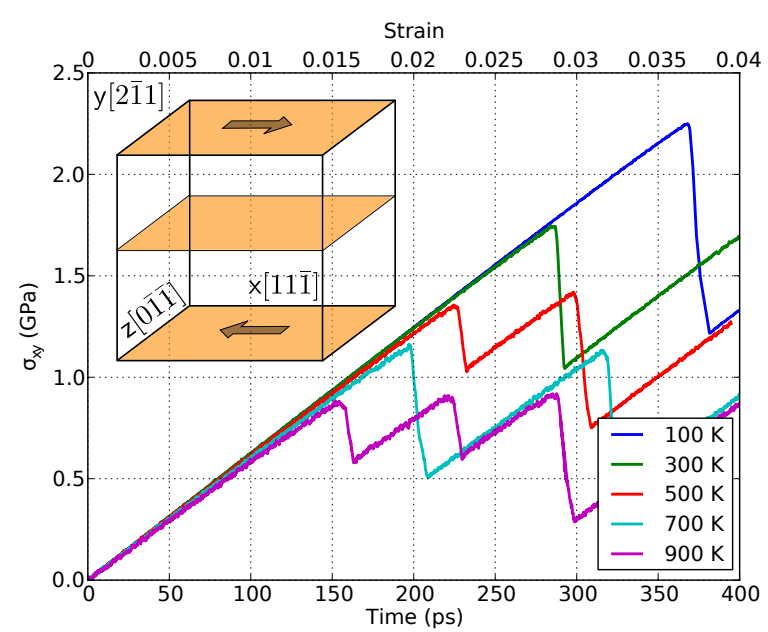

a)

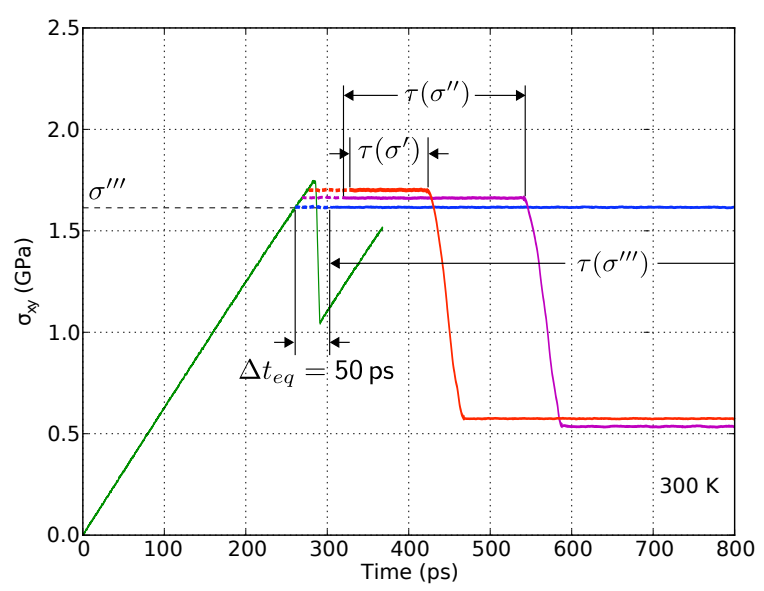

b)

FIG. 1. Schematics of the modeling: the shear stress $\sigma_{x y}$ is applied along the $(2 \overline{1} 1)$ plane in the [11 $\overline{1}]$ direction - the inset in (a). Two modes of stress application are shown in (a) and (b). 1a) In the constant shear rate $\left(10^{8} \mathrm{~s}^{-1}\right)$ mode, the stress rises linearly in the elastic regime and peaks at the onset of plastic deformation. The resulting sawtooth stress behavior is shown in (a) for different temperatures. 1b) To simplify the analysis, a constant stress mode is used to find the nucleation rates. A sawtooth curve from above (green) is displayed for reference. Its approximate peak stress locates the region of rapid nucleation on MD timescales. Three examples of $\sigma_{x y}=$ const. are shown at nearby stresses (blue, magenta, red). Instantaneous atomic coordinates from the constant strain rate simulation are used as initial conditions. Each constant stress case is subjected to an equilibration procedure (labeled for blue). Only two cases show a nucleation event in the plot; lower stresses lead to longer nucleation times on average.

each temperature, the dimensions of the simulation cell were fully relaxed.

Our goal is to obtain the loop nucleation rates under stationary conditions, during which the system is held at constant temperature and strain (this corresponds to 
constant stress, as there are no mobile defects in the system to mediate plastic deformation prior to the nucleation of a partial loop). In order to locate the conditions for timely nucleation, the equilibrated system was subjected to simple shearing along the $[11 \overline{1}]$ direction at a constant strain rate of $10^{8} \mathrm{~s}^{-1}$ for each temperature. As in the previous step, the temperature was controlled via a strong Langevin thermostat of $0.1 \mathrm{ps}$. The results are shown in Fig. 1 (a). In the elastic region there is a linear increase of stress with strain. The nucleation of a twinning loop converts elastic to plastic strain. This is seen as a drop of the stress vs. strain curve with the average yield point dependent on temperature. The loop grows rapidly enough that the stress falls until the new layer is completed across the periodic boundary conditions. Subsequently, the stress increases again and another nucleus may form on either coherent interface, giving the sawtooth behavior. A feature of our simulations is that the size of the simulation box and timescales are such that there is at most only one stable loop per interface at any given time, as the release of local stress determined by its rapid growth is enough to suppress any additional loop formation.

Once the threshold is located, we select instantaneous atomic configurations corresponding to systems with subcritical strains, taken from times in Fig. 1 (b). The original constant strain rate simulation is again shown in green $(300 \mathrm{~K})$. Static strain configurations (red, magenta, blue) are also shown, selected from points prior to the first onset of plasticity in the green curve at the sawtooth instability. Higher stress leads to more rapid nucleation on average. The hydrostatic pressure is slightly elevated at these strains, mainly due a change in the stress component normal to the twinning plane with value of -0.15 GPa. This relatively small stress is ignored. Instantaneous atomic configurations from the constant strain-rate simulation are then employed as initial states for fixed strain simulations, where loop nucleation will occur at a greatly reduced rate. In order to reduce any transient effect from the dynamic ramp in stress in the preceding stage, the samples were held at constant strain, quenched to temperatures of $1 \mathrm{~K}$, and then returned to the desired temperature over $\Delta t_{e q}=50 \mathrm{ps}$, using Langevin thermostats with decay times of 1 ps and 10 ps for the cooling and heating processes, respectively.

Finally, for each fixed strain value we selected $N=50$ independent replicas that remained loop-free during their thermal cycles. Subsequently, the temperature control is turned off, and the system evolved in the micro-canonical ensemble until a nucleation event occurs. We define the time to first nucleation for the $i$-th replica, $\tau_{i}$, as the time for which the MD stress, $\sigma_{x y}$, remains within seven standard deviations of the average value for loop-free systems. The nucleation time for each replica is then an independent random variable from some probability distribution characteristic of the system conditions and sample preparation regimen. We estimate the loop nucleation rate from this sampling. As there may still be an initial interval during which the sample preparation history perturbs the nucleation process, we applied two methods, described in the next section, in order to eliminate transient effects.

\section{RESULTS AND DISCUSSION}

\section{A. Direct method: stationarity test}

The mean time to first nucleation for a stationary distribution of loop-free systems at constant temperature and strain should be independent of when we begin measurement. To get reliable estimates from our ensemble as prepared in Sec. II, we impose a stationarity test for a delay-dependent mean nucleation time, $\bar{\tau}\left(t_{0}\right)$. We choose a sub-sample of the $N$ original replicas, $i=1, n$, with $\tau_{i} \geq t_{0}$ (i.e., we discard any simulations which nucleate prior to $\left.t_{0}\right)$. Then the estimated lifetime is:

$$
\bar{\tau}\left(t_{0}\right):=\frac{\sum_{i\left(\tau_{i} \geq t_{0}\right)}^{N}\left(\tau_{i}-t_{0}\right)}{n\left(\tau_{i} \geq t_{0}\right)},
$$

with $n\left(\tau_{i} \geq t_{0}\right)$ the number of replicas with $\tau_{i} \geq t_{0}$. Fig. 2 shows the results of this approach for a case at $300 \mathrm{~K}$. Finally, we define an average-window criterion for $t_{0}$, within which $\bar{\tau}\left(t_{0}\right)$ is stable. Estimates for $\bar{\tau}$ using low values of $t_{0}$ are discarded, as they may include residual transient behavior, while high values of $t_{0}$ are also avoided, because the small surviving population gives poor statistics. Within the broad acceptance window, the selected nucleation times are consistent with the Poisson distribution, i.e. the loop-free population decays exponentially with time, and the estimated mean or half-life then provides the nucleation rate.

\section{B. Mean first-passage time}

An alternative way to get nucleation rates is based on the concept of mean first-passage time (MFPT) ${ }^{15,19}$, which is defined as the average time $\tau(n)$ that a cluster spends to reach, for the first time, a given size $n$. For sufficiently high nucleation barrier, $\tau(n)$ can be written $\operatorname{as}^{15}$

$$
\tau(n)=\frac{\tau_{J}}{2}\left(1+\operatorname{erf}\left(\sqrt{\pi} Z\left(n-n^{*}\right)\right)\right)
$$

where $\tau_{J}$ is the nucleation time, $n^{*}$ is the critical cluster size, $Z$ is the Zeldovich factor, and the error function $\operatorname{erf}(\mathrm{x})=(2 / \sqrt{\pi}) \int_{0}^{\mathrm{x}} \mathrm{e}^{-\mathrm{t}^{2}} \mathrm{dt}$. In the context in which we are working, we consider a cluster as a set of atoms bounded by the twinning dislocation loop, which are selected by using the centrosymmetry parameter ${ }^{20}$. The cluster-size distribution in the system was monitored every 1 ps, using the Stoddard algorithm ${ }^{21}$ with a cutoff 


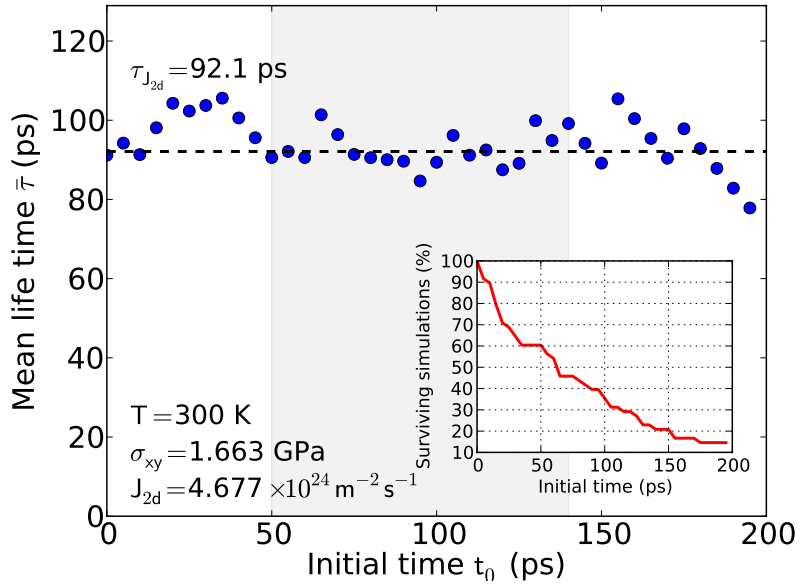

FIG. 2. Estimated (mean) lifetime to loop nucleation, $\tau_{J 2 d}$, versus sample delay-time, $t_{0}$. The stability of results taken from the wide interval marked in grey indicates that the initial sampling is adequately-converged. The inset shows the number of surviving replicas versus time. The curve approximates an exponential decay for samples starting inside the acceptance window.

radius of $1.5 a_{0}$, with $a_{0}$ the lattice constant. In Fig. 3 we show the MFPT as a function of the maximum cluster size for the case corresponding to Fig. 2. A fit to the Eq. 7 gives us a nucleation time of $\tau_{J_{2 d}}=92.9 \mathrm{ps}$, in very close agreement with the result obtained via the stationarity test, but with a smaller statistical error thanks to its well defined plateau for larger values of the nucleation size $n$. MFPT method also provides information about the critical cluster size, which is given by the inflection point in Fig. 3. For $300 \mathrm{~K}$ and $1.663 \mathrm{GPa}$ the critical cluster size is $\sim 12 \pm 1$ atoms (see insets on Fig. 3). The critical nucleus is a small perturbation on the entire system, occupying $\sim 0.1 \%$ of the interface area and $\sim 0.001 \%$ of the total cell volume. In order to determine the shape of the critical cluster, we fitted the distribution of atoms (averaged over all the replicas) to an ellipse, which gave us an eccentricity of $0.94 \pm 0.5 \%$. The ellipse's major axis is oriented along the close-packed [111] direction that is the most probable for local atomic displacement under the applied shear.

\section{Nucleation rates: dependency on driving force and temperature}

The (steady-state) nucleation rate is given by $J_{2 d}=$ $1 /(2 \tau A)$, where $A$ is the area of one twin boundary in the simulation cell, $\tau$ is the mean nucleation time in the given cell, and the factor of 2 corresponds to the number of coherent interfaces. In Fig. 4 we show a few nucleation rates calculated with system at a fixed strain value of $\epsilon_{x y}=0.0255$ for some temperatures in the interval 300-320 K. The slope of the curve gives the value of the

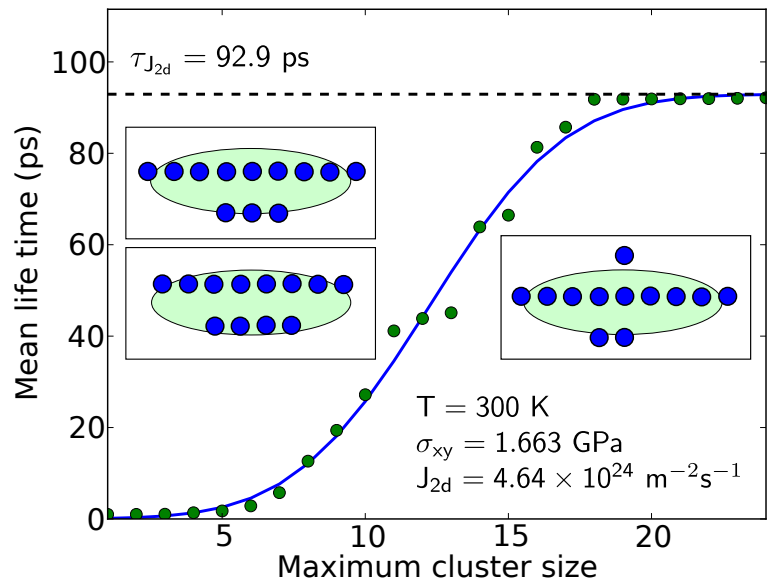

FIG. 3. Mean first-passage times (MFPT) corresponding to the case shown in Fig. 2. At $300 \mathrm{~K}$ and $1.663 \mathrm{GPa}$ the critical cluster size is $12 \pm 1$ atoms. Insets: some of the critical cluster shapes observed in the simulations. The ellipse (eccentricity of $\sim 0.94$ ) was obtained after averaging on the replicas. The major axis is oriented parallel to the close-packed direction $[11 \overline{1}]$.

activation energy at this particular strain ${ }^{9}, 0.97 \mathrm{eV}=$ $1.55 \times 10^{-12} \mathrm{erg} \pm 5 \%$, and a line energy $\gamma_{d}=0.035 \mathrm{eV} / \AA$ $=5.62 \times 10^{-6} \mathrm{erg} / \mathrm{cm} \pm 5 \%$. The exponential prefactor contains an entropic contribution, formally defined as the reduction rate of the activation free energy with increasing temperature ${ }^{9}$. Using the Debye frequency for tantalum $\left(5 \times 10^{12} / \mathrm{s}\right)^{22}$ for the frequency prefactor , and considering the atomic density on the (21) plane $(7.44$ $\left.\times 10^{18} \mathrm{~m}^{-2}\right)$, an estimate of the entropic contribution in the constant strain ensemble is $\sim 18 k_{B}$.

We performed a consistency check on the activation energy by quenching a nearly critical cluster with 15 atoms. This was done by iteratively freezing and relaxing the cluster and the rest of the system in order to remove thermal disorder while preventing the collapse of the cluster at lower temperatures. The activation energy is given by $E_{c}=E\left(n_{c}\right)-E(0)$, where $E\left(n_{c}\right)$ and $E(0)$ denote the system energies for the configuration with and without the cluster ${ }^{9}$. This approach gives us an activation energy of $0.87 \mathrm{eV}=1.40 \times 10^{-12} \mathrm{erg}$. An additional comparison can be made by considering the energy of a circular dislocation loop ${ }^{7}$. For a core radius of 2 times the Burgers vector for the twin dislocation $(\sim 2 \AA)$, we get $1.05 \mathrm{eV}=$ $1.69 \times 10^{-12} \mathrm{erg}$, which provides additional confidence in our results.

In some applications it is desirable to have the nucleation rate as a function of stress for a fixed temperature, e.g. when an experiment provides a stress history. In Fig. 5 we show a few nucleation rates calculated with system at $300 \mathrm{~K}$, as a function of the inverse of the driving force $\Delta \mu$. Because this set of simulations contains results for slightly different stress values, it is not easy to uniquely identify the activation energies and entropic 


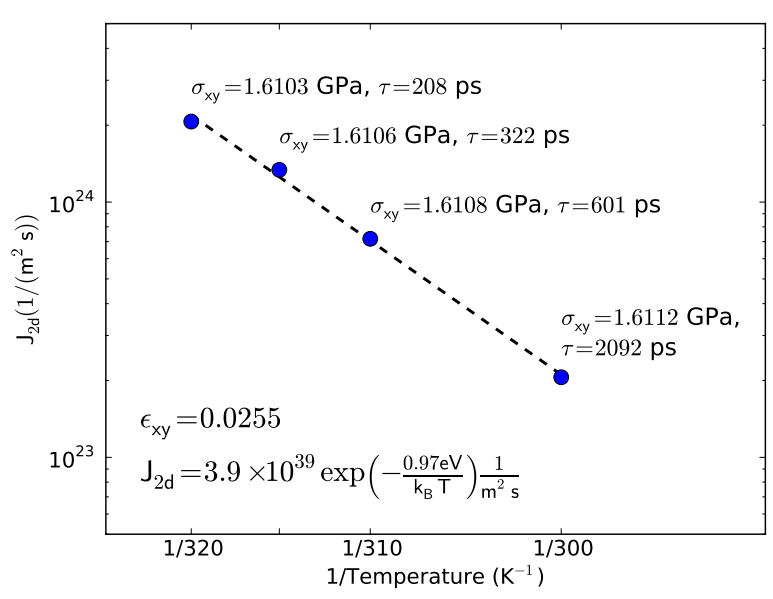

FIG. 4. Homogeneous nucleation rate for a partial dislocation loop on the Ta $(2 \overline{1} 1)$ coherent twin interface, as a function of temperature at fixed strain of $\epsilon_{x y}=0.0255$. The circles indicate the results of MD simulations, and the dashed line corresponds to a fit using Eq. 4.

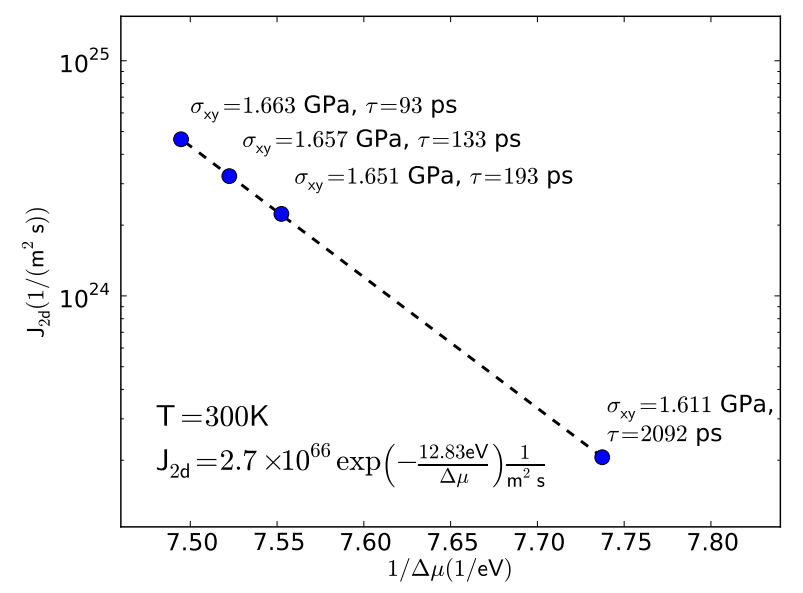

FIG. 5. Homogeneous nucleation rate for a partial dislocation loop on the Ta (2111) coherent twin interface, as a function of the inverse driving force for $300 \mathrm{~K}$. The circles indicate the results of MD simulations, and the dashed line corresponds to a fit using Eq. 4.

contributions. A direct fit to this data gives a large exponential prefactor of $2.7 \times 10^{66} \mathrm{~m}^{-2} \mathrm{~s}^{-1}$, counterbalanced by a high activation energy of $12.83 \mathrm{eV}$ in the exponential function. The dramatic difference from the constant strain case is to be expected; it has already been established that the entropic contribution in a constant stress ensemble is significantly higher than the corresponding entropic contribution in the constant strain ensemble ${ }^{8,9}$.

In Fig. 6 we locate some of the nucleation rates obtained in this work on a plot of $\Delta \mu$ versus temperature. Due to computational restrictions, direct MD simulations can only be used to study the nucleation process on a

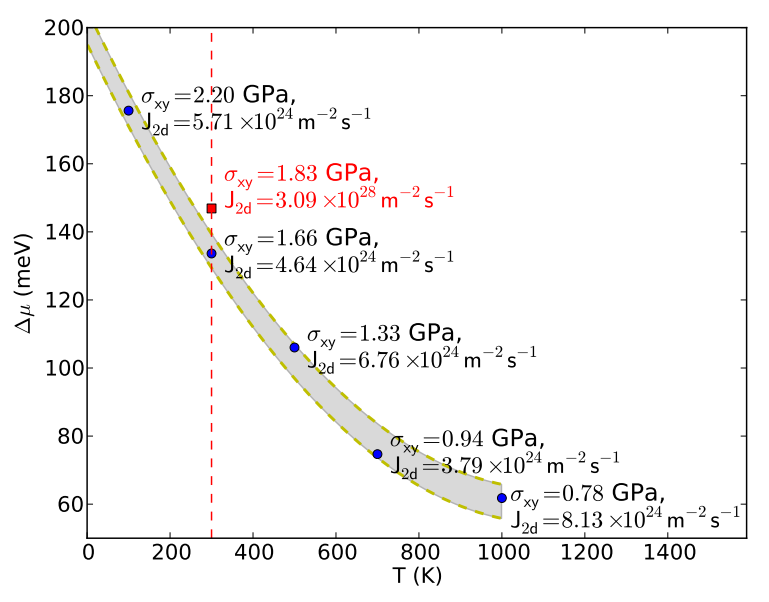

FIG. 6. Homogeneous nucleation rates for partial dislocation loops on the Ta $(2 \overline{1} 1)$ coherent twin interface obtained via direct MD simulations. The parameters at which nucleation is accessible to direct MD simulations are sketched as a narrow band, as found using the procedure described in Fig. 1. Typical experiments fall below this region ${ }^{23}$. Higher stress and driving force, $\Delta \mu$, imply an increasing contribution from nucleation of twinning dislocation loops as compared to the pole mechanism. A red square marks one point at which the twin growth rate by the nucleation of partial dislocations would equal the one dictated by the pole mechanism. These conditions are relevant to some more dynamic experiments ${ }^{24,25}$. Heterogenous loop nucleation generally will be faster than the homogeneous mechanism shown here and may be experimentally relevant at even lower temperature and driving force.

timescale of a few nanoseconds. Cases at which nucleation is practically achievable are sketched as a narrow band in Fig. 6, wherein the nucleation rate is approximately constant. Below this band, the nucleation is too slow to access in an unaccelerated MD simulation, while above the nucleation time is short compared to the time to reach a quasistationary ensemble of replicas at the given strain. Typically, experiments occur at relatively low strain rates; dislocation mediated plasticity can accommodate the deformation, and the resulting stress is low. Such experiments lie below this MD-accessible band.

For moderate to high stresses like those in Fig. 6, the partial dislocation velocity is expected to saturate near the shear wave sound velocity. This maximum is $v_{t} \sim 2000 \mathrm{~m} / \mathrm{s}$ for this Ta potential. We can now estimate a stress at which the migration rate of the coherent twin interface due to the nucleation and growth of twinning dislocations Eq. 5 begins to exceed the corresponding growth velocity due to the pole mechanism under similar thermodynamic conditions, as given by ${ }^{14}$.

$$
v_{p}=\frac{h v_{t}}{4 \pi r_{c}}
$$

with $r_{c}=\omega \gamma_{d} / h \Delta \mu$ the radius of the two-dimensional critical nucleus. This expression follows from the simplest 
relationship for a single screw dislocation, as the velocity may vary considerably for a complex extended source, especially at a high driving force (the "self-interaction" of partial dislocations in the spiral is stronger at high stresses, as $r_{c}$ and the spacing between neighboring spiral turns decrease). At $300 \mathrm{~K}$, the two growth mechanisms rates reach parity for a shear stress of $1.8 \mathrm{GPa}$, at $v_{\text {nuc }}=$ $v_{\text {pole }} \simeq 67 \mathrm{~m} / \mathrm{s}$. Assuming isotropy and uniaxial strain ${ }^{26}$, such stress corresponds to a normal stress of $\sim 7.5 \mathrm{GPa}$, which is attainable at high strain rate conditions, as in gas-gun plate impact experiments ${ }^{24,26}$, and laser-driven compression $^{27}$. The corresponding conditions have been highlighted in Fig. 6 (red square).

\section{CONCLUSIONS}

By using a molecular dynamics approach we have successfully calculated a set of nucleation rates of twinning dislocation loops for Tantalum at different temperatures. We have applied a stationarity test and the mean firstpassage time (MFPT) method in order to get nucleation times. Although both methods provide similar nucleation times, the MFPT method presents significant advantages as it considers the kinetics of the process, providing additional information (critical size of the twinning dislocation loop, location of the barrier, etc.)

High strain rate or low temperature situations can reach the kinds of stresses seen in Fig. 6. In such cases, significant contributions to twin growth from a KJMA nucleation and growth mechanism can be expected. If the detailed stress history and final microstructure of a sample is available, then it will be possible to compare the predicted twin size to observed microstructure.

An estimate of the contribution to the twinning growth dictated by the $2 \mathrm{~d}$-nucleation mechanism described here can be obtained by considering some existing experimental results ${ }^{24}$. Reed et al. have derived strength information from gas-gun-driven plate impact experiments in Tantalum. Specifically, we have considered the experiment denoted as "shot 1576", characterized by a peak pressure of $\sim 10 \mathrm{GPa}$, and peak deviatoric stress (or peak shear stress, here taken as $\sigma_{x y}$ ) and strain rate of $\sim 0.9 \mathrm{GPa}$ and $2 \times 10^{6} / \mathrm{s}$, respectively, within an interval of high stress of $0.5 \mu \mathrm{s}$. By using their deviatoric stress histories and integrating the growth rate, Eq. 5, over this time interval, we get a negligible average twin thickness from nucleation and growth. Although a microstructural analysis was not included in Ref. 24, we could predict, by considering Eq. 8, a final average thickness of $\sim 40 \mu \mathrm{m}$. This suggests that twins formed in those experiments grew exclusively by the pole mechanism. It is interesting to note that if the deviatoric stress history had peak values of $2 \mathrm{GPa}$ (over the same time interval), twin grown by 2 d-nucleation of twinning dislocation loops would also have average thickness values of $\sim$ $40 \mu \mathrm{m}$. This gives us an idea of the conditions under which exists a strong competition of these two twinning growth mechanisms. However, the comparison may be complicated by the possibility of other models for coherent boundary growth. This will be studied in a future publication. Additionally it is worth pointing out that interactions with the microstructure, which are not being considered here, could limit the twin growth in real materials.

The order of magnitude of nucleation rates reachable via direct MD simulation is $\sim 10^{24} \mathrm{~m}^{-2} \mathrm{~s}^{-1}$, which defines a narrow band of nucleation rates in the driving force vs. temperature plot. Adoption of an accelerated molecular dynamics scheme would be advantageous ${ }^{7,8}$ in order to extend the range of of nucleation rates by orders of magnitude. This would allow to study the validity of the Eq. 4, and would provide improved parameters for twinning constitutive models. This is also a subject of ongoing study.

\section{ACKNOWLEDGMENTS}

The authors gratefully acknowledges Robert Rudd, Mukul Kumar, Nathan Barton, Jim Belak, Kyle Caspersen, Vasily Bulatov and Frederick Streitz for their contributions and fruitful discussions. This work was performed under the auspices of the U.S. Department of Energy by Lawrence Livermore National Laboratory under Contract DE-AC52-07NA27344 (LLNL-JRNL-638982).
* Currently at Theoretical Division T-1, Los Alamos National Laboratory, Los Alamos, NM 87545, USA.

1 S. V. Lubenets, V. I. Startsev, and L. S. Fomenko, Physica Status Solidi (A) 92, 11 (1985).

2 J. W. Christian and S. Mahajan, Prog. Mater. Sci. 39, 1 (1995).

3 Y. T. Zhu, X. Z. Liao, S. G. Srinivasan, Y. H. Zhao, M. Baskes, F. Zhou, and E. J. Lavernia, Appl. Phys. Lett. 85, 5049 (2004).

4 M. Y. Gutkin, I. A. Ovid'ko, and N. V. Skiba, Phys. Rev. B 74, 172107 (2006).

${ }^{5}$ T. Zhu, J. Li, S. Ogata, and S. Yip, MRS Bulletin 34, 167
(2009).

6 K. Lagerlof, J. Castaing, P. Pirouz, and A. H. Heuer, Philosophical Magazine A 82, 2841 (2002).

7 S. Aubry, K. Kang, S. Ryu, and W. Cai, Scr. Mater. 64, 1043 (2011).

8 S. Ryu, K. Kang, and W. Cai, Proc. Natl. Acad. Sci. U. S. A. 108, 5174 (2011).

9 S. Ryu, K. Kang, and W. Cai, J. Mater. Res. 26, 2335 (2011).

10 A. N. Kolmogorov, Bull. Acad. Sci. USSR, Math Ser. 3, 355 (1937).

11 W. A. Johnson and P. A. Mehl, Trans. Am. Inst. Min., 
Metall. Pet. Eng. 135, 416 (1939).

12 M. Avrami, J. Chem. Phys. 7, 1103 (1939).

13 M. Abramowitz and I. A. Stegun, Handbook of Mathematical Functions: with Formulas, Graphs, and Mathematical Tables, edited by M. Abramowitz and I. A. Stegun (Dover Publications, New York, 1965).

14 A. A. Chernov, E. I. Givargizov, K. S. Bagdasarov, V. A. Kuznetsov, L. N. Demianets, and A. N. Lobachev, Modern Crystallography III, edited by A. A. Chernov (SpringerVerlag, Berlin, 1984) p. 131.

15 J. Wedekind, R. Strey, and D. Reguera, J. Chem. Phys. 126, 134103 (2007).

16 G. J. Ackland and R. Thetford, Philosophical Magazine A 56, 15 (1987).

17 P. Bristowe and A. Crocker, J. Phys. F: Metal Phys. 4, 1859 (1974).

18 Y. Mishin and A. Lozovoi, Acta Mater. 54, 5013 (2006).

19 P. Hänggi, P. Talkner, and M. Borkovec, Rev. Mod. Phys.
62, 251 (1990).

20 C. L. Kelchner, S. J. Plimpton, and J. C. Hamilton, Phys. Rev. B 58, 11085 (1998).

21 S. D. Stoddard, J. Comput. Phys. 27, 291 (1978).

22 C. Kittel, Introduction to solid state physics (WileyInterscience, New York, 1986).

${ }^{23}$ N. R. Barton, J. V. Bernier, R. Becker, A. Arsenlis, R. Cavallo, J. Marian, M. Rhee, H.-S. Park, B. Remington, and R. T. Olson, J. Appl. Phys. 109, 073501 (2011).

24 B. W. Reed, J. Reed Patterson, D. C. Swift, J. S. Stolken, R. W. Minich, and M. Kumar, J. Appl. Phys. 110, 113506 (2011).

25 V. Livescu, J. F. Bingert, and T. A. Mason, Mater. Sci. Eng. A 556, 155 (2012).

26 G. T. Gray III, N. K. Bourne, and J. C. F. Millet, J. App. Phys. 94, 6430 (2003).

27 C. H. Lu, B. A. Remington, B. R. Maddox, B. Kad, H. S. Park, S. T. Prisbrey, and M. A. Meyers, Acta Mater. 60, 6601 (2012). 